\title{
Image detection of yeast Saccharomyces cerevisiae by light-addressable potentiometric sensors (LAPS)
}

Zhang, DW; Wu, F; Wang, J; Watkinson, M; KRAUSE, SK

(C) 2016 Elsevier B.V.

http://dx.doi.org/10.1016/j.elecom.2016.08.017

For additional information about this publication click this link.

http://qmro.qmul.ac.uk/xmlui/handle/123456789/15471

Information about this research object was correct at the time of download; we occasionally make corrections to records, please therefore check the published record when citing. For more information contact scholarlycommunications@qmul.ac.uk 


\title{
Image detection of yeast Saccharomyces cerevisiae by light-addressable potentiometric sensors (LAPS)
}

De-Wen Zhang ${ }^{\mathrm{a}, \mathrm{c}}$, Fan Wu ${ }^{\mathrm{a}}$, Jian Wang ${ }^{\mathrm{a}}$, Michael Watkinson ${ }^{\mathrm{b}}$, Steffi Krause ${ }^{\mathrm{a},{ }^{*}}$

${ }^{a}$ School of Engineering and Materials Science, Queen Mary University of London, Mile End Road, London, E1 4NS, UK

${ }^{\mathrm{b}}$ School of Biological and Chemical Sciences, Queen Mary University of London, Mile End Road, London, E1 4NS, UK

${ }^{\mathrm{c}}$ China Academy of Engineering Physics, P.O. Box 919-71, Mianyang, 621900, Sichuan, P.R. China

\begin{abstract}
A light-addressable potentiometric sensor (LAPS) was developed for the image detection of multilayer yeast Saccharomyces cerevisiae. By modifying silicon-on-sapphire (SOS) with a monolayer of 1,8-nonadiyne, an inert LAPS substrate, insensitive to $\mathrm{pH}$ was obtained. Low gelling temperature agarose gel was shown to be an effective immobilization reagent for yeast cells in LAPS measurements without causing a background signal. The photocurrent images obtained were shown to be induced by the surface negative charge and the local impedance of the yeast cells.
\end{abstract}


Keywords: LAPS, Scanning Photo-induced Impedance Microscopy (SPIM), Cell imaging, Saccharomyces cerevisiae

\section{Introduction}

Live-cell imaging methods that have been used to investigate the structural organization and dynamic processes of living cells or tissues non-invasively comprise optical microscopies and electrochemical imaging methods. Optical microscopy, especially fluorescence microscopy, is most widely performed in live-cell imaging for the study of the physiological state of cells, cellular transport or cell growth [1]. The development of super-resolved fluorescence microscopy has brought optical microscopy into the nanodimension bypassing Abbe's diffraction limit and has enabled the visualisation of the pathways of individual molecules such as proteins inside living cells with the help of fluorescent molecules [2, 3]. Electrochemical imaging methods are alternative techniques for live-cell imaging, including scanning electrochemical microscopy (SECM) [4, 5], scanning ion conductance microscopy (SICM) [6, 7], plasmonic-based electrochemical impedance microscopy (EIM) [8], and lightaddressable potentiometric sensors (LAPS) [9]. They can map parameters such as Faradaic current, ion conductivity, local impedance, extracellular potentials and charges, which cannot be measured with optical microscopy. SECM maps local electrochemical current by scanning a microelectrode across the surface, while the SICM maps the ion conductance by scanning a micropipette. Plasmonic-based EIM monitors local impedance by measuring the surface plasmon resonance (SPR) response to an applied ac signal. LAPS measures the local photocurrent at electrolyte/insulator/semiconductor field-effect structures in response to the Nernst potential at the electrolyte/insulator interface by illuminating the semiconductor with a focused, modulated laser beam without the need for scanning microtips or labels, which would be highly advantageous for the non-invasive imaging of cells.

LAPS has been used successfully for the electrochemical imaging of specific ions, DNA detection, monitoring of enzymatic reactions, microorganisms, activities and extracellular potentials of cells [10-16] with a typical resolution of about 10-100 $\mu \mathrm{m}$ using LED arrays as the light source. Using an optimized laser scanning setup in conjunction with a silicon-onsapphire (SOS) substrate (Figure 1), $1.5 \mu \mathrm{m}$ spatial resolution was obtained using visible light with a wavelength of $405 \mathrm{~nm}$, and $0.8 \mu \mathrm{m}$ resolution was achieved using light with energy smaller than the bandgap exciting a two-photon effect in silicon $[17,18]$. To improve the sensitivity of the LAPS surface, the traditional insulator was replaced with a 1,8-nonadiyne 
monolayer that was bound to hydrogen terminated silicon on SOS $[19,20]$. The technique has been adapted to the imaging of the local impedance, which has been termed Scanning photoinduced impedance microscopy (SPIM) [21-23].

In this work, we immobilized yeast Saccharomyces cerevisiae on a 1,8-nonadiyne modified SOS surface using low gelling temperature agarose gel. The LAPS images of multilayer yeast cells were recorded using a diode laser (405 nm) with good lateral resolution.

\section{Experimental}

\subsection{Materials}

Silicon-on-sapphire (SOS) with a $1 \mu \mathrm{m}$ thick silicon (100) layer (boron doped, $0.1 \Omega \mathrm{cm}$ ) on a $475 \mu \mathrm{m}$ thick sapphire substrate was purchased from Monocrystal, Russia. Yeast Saccharomyces cerevisiae was purchased from a local supermarket. All chemicals were purchased from Sigma-Aldrich without further purification unless noted. 1,8-Nonadiyne (98\%) was redistilled before use as reported [23]. All samples and buffer solution were prepared using ultrapure water $(18.2 \mathrm{M} \Omega \mathrm{cm})$ from a Milli-Q water purification system (Millipore, USA).

\subsection{Preparation of 1,8-nonadiyne modified SOS}

The SOS substrate was modified with an ohmic contact and subsequently with 1,8-nonadiyne as described previously [23]. After modification, the SOS substrate was cut into $4 \mathrm{~mm} \times 4$ $\mathrm{mm}$ pieces before use.

\subsection{Immobilization of yeast cells}

Low gelling temperature agarose gel was used to immobilize cells onto the SOS surface. After boiling for $5 \mathrm{~min}$, the $1.5 \%$ agarose gel was slowly cooled down to $37^{\circ} \mathrm{C}$, and was then held at $37{ }^{\circ} \mathrm{C}$ in a compact dry bath and a block heater (Thermo Scientific). Yeast was washed five times in ultrapure water by centrifuging (1800 rpm, $5 \mathrm{~min})$. The cells were kept in pure water to prevent any interference and used immediately after washing. To check the viability of the yeast cells after the preparation, the $\mathrm{pH}$ shift of the $4 \mathrm{mg} / \mathrm{mL}$ yeast in $10 \mathrm{mM}$ $\mathrm{pH}$ 7.4 PBS solution containing $5 \mathrm{mM}$ glucose was measured with time. The $\mathrm{pH}$ decreased from 6.93 to 6.34 in the first two hours due to extracellular acidification, and then remained constant in the next 43 hours. The agarose gel was mixed with $40 \mathrm{mg} / \mathrm{mL}\left(4 \times 10^{8}\right.$ cells $\left./ \mathrm{mL}\right)$ yeast (v/v, 9:1). One drop of the mixture $\left(\sim 0.2 \mu \mathrm{L}, 4 \times 10^{7}\right.$ cells $\left./ \mathrm{mL}\right)$ was added onto the 1,8 - 
nonadiyne modified SOS surface. The yeast-agarose gel formed after it slowly cooled down to room temperature effectively immobilizing a multilayer of yeast cells on the 1,8-nonadiyne modified SOS surface.

\subsection{LAPS measurements}

The setup for LAPS and SPIM measurements was described previously [17]. A diode laser LD1539 (Laser 2000, $\lambda=405 \mathrm{~nm}, 1 \mathrm{~mW}$ ) was used for photocurrent excitation. The modulation frequency was $1 \mathrm{kHz}$. AC photocurrents were measured by an EG\&G 7260 lockin amplifier using a platinum electrode and an $\mathrm{Ag} / \mathrm{AgCl}$ electrode as the counter and reference electrodes. The lateral resolution of the LAPS setup at a laser wavelength of 405 $\mathrm{nm}$ and using SOS substrates was determined to be $1.5 \mu \mathrm{m}$ as described previously $[17,18]$.

\subsection{Impedance measurements}

AC impedance measurements were carried out in PBS using an Autolab PGSTAT30/FRA2 (Windsor Scientific Ltd., UK) using a three-electrode system (see 2.4), a potential of $0 \mathrm{~V}$ and an amplitude of $5 \mathrm{mV}$.

\section{Results and Discussion}

The water contact angle $\left(84 \pm 1^{\circ}\right)$ for the 1,8-nonadiyne modified SOS surface was measured by a Drop Shape Analysis System (Krüss DSA100, Germany), which indicated a hydrophobic monolayer and agreed with results reported previously [20]. Photocurrent measurements using 1,8-nonadiyne modified SOS surfaces displayed the expected behaviour for good quality LAPS substrates. LAPS have been developed for the determination of the extracellular acidification of Escherichia coli and Saccharomyces cerevisiae using $\mathrm{pH}$ sensitive surfaces in past reports $[12,14,24]$. In this report, we were aiming to detect the properties of cells themselves to obtain a common imaging method, which can be widely used for other cell types in the future. Hence, an alkyne terminated monolayer was chosen in our experiments to exclude the interference of possible extracellular acidification by the metabolic activity of yeast cells. To confirm that the sensor surface was not $\mathrm{pH}$ sensitive, the LAPS responses of 1,8-nonadiyne modified SOS surfaces and that covered with agarose gel were measured in a series of $\mathrm{pH}$ phosphate buffer solutions ( $\mathrm{pH}$ 5.5-7.9) with an interval of about $0.5 \mathrm{pH}$ units. As shown in Figure 2, no significant surface potential shift of the photocurrent-voltage curves with $\mathrm{pH}$ was detected. The results conformed to the theoretical 
response of the alkyne-terminated monolayer modified SOS surface without proton selectivity. To investigate the stability of the surface after exposure to different $\mathrm{pH}$ values, photocurrent-voltage curves of the agarose gel covered surface were measured before and after exposure to $\mathrm{pH} 7.3$ and $\mathrm{pH} 5.5$ phosphate buffers for 24 hours. There was no significant shift of the normalized $I-V$ curves.

A multilayer of yeast cells $\left(\sim 0.2 \mu \mathrm{L}, 4 \times 10^{7}\right.$ cells $\left./ \mathrm{mL}\right)$ was immobilized on the 1,8 nonadiyne modified SOS surface by low gelling temperature agarose gel (Figure 3A(b)). Pure agarose gel was also dropped onto the same sample surface as a control experiment (Figure 3A(a)). Figure 3B shows the corresponding LAPS image of yeast-agarose gel on a 1,8nonadiyne modified SOS surface at $0.7 \mathrm{~V}$ using a $405 \mathrm{~nm}$ laser. The step size was $5 \mu \mathrm{m}$. The scanning time was about $15 \mathrm{~min}$. As a control experiment, pure agarose gel was deposited on the sample (area (a) in Figures 3A and B). The agarose gel coated area showed the same photocurrent as the blank 1,8-nonadiyne modified SOS and is therefore not visible on the photocurrent image in Figure 3B. This is also confirmed by the identical photocurrent curves obtained for the agarose gel coated area and the blank 1,8-nonadiyne modified SOS shown in Figure $3 \mathrm{C}$. Due to its porous structure, the agarose gel allows conductive ions and small molecules to penetrate the gel freely, which indicated that it provided an ideal immobilization method for yeast cells in LAPS measurements. As shown in Figure 3C, the maximum photocurrent in the area where yeast cells were attached was about 15\% lower than that on the blank monolayer surface and the agarose gel coated area, indicating an increase of the local impedance. After normalizing, the $I-V$ curves (Figure 3D) on the yeast attached area showed a shift of about $+90 \mathrm{mV}$ compared to the blank monolayer surface and the agarose gel attached area, corresponding to the negative surface charge of the yeast cells in $\mathrm{pH} 7.4$ PBS solution [25].

After removing the yeast-agarose gel on the 1,8-nonadiyne modified SOS surface by water flow, the LAPS image of yeast-agarose gel disappeared, which further proved that the LAPS signals in Figure 3 were produced by the yeast cells.

To verify if the LAPS signals of the yeast cell were not produced by the yeast cells' secretion, the supernatant of the $40 \mathrm{mg} / \mathrm{mL}$ yeast cells' centrifugate mixed with the agarose gel was detected by the same procedure. The result indicated that there was no significant change between the supernatant-agarose attached SOS surface and the blank 1,8-nonadiyne modified SOS surface. Therefore, the LAPS signal was produced by the surface charge and impedance of yeast cells but not the yeast cells' secretion. 
The complex local impedance can be determined from the maximum photocurrent measured in inversion - a technique that has been termed SPIM [21-23]. The impedance data obtained from SPIM and from classical AC impedance measurements were compared. Figure 3E shows the maximum photocurrents measured at a dc bias of $1.0 \mathrm{~V}$ on the blank monolayer surface and a yeast modified area at different modulation frequencies. The maximum photocurrent decreased on the yeast coated area. The expression for the impedance and the maximum photocurrent is given in Equation 1:

$\frac{V_{\text {photo }}}{I_{\text {photo }}}=Z$

where $V_{\text {photo }}$ is the photovoltage, $I_{\text {photo }}$ is the maximum photocurrent, $Z$ is the impedance of the sample.

Assuming that the photovoltage is the same, the impedance of the yeast modified area can be estimated from

$Z_{\text {yeast }}=Z_{\text {nona }} \frac{I_{\text {nona }}}{I_{\text {yeast }}}$

where $Z_{\text {yeast }}$ is the impedance of the yeast attached surface, $Z_{\text {nona }}$ is the impedance of the 1,8 nonadiyne modified SOS surface obtained from AC impedance spectroscopy.

Figure $3 \mathrm{~F}$ shows the AC impedance spectra for the blank 1,8-nonadiyne modified surface, a surface entirely covered with the entire yeast-agarose gel and an impedance spectrum of a yeast-agarose gel coated area derived from local photocurrent measurements using Equation 2. The impedance increased after the immobilization of the yeast-agarose gel. The values for $Z_{\text {yeast }}$ calculated from local photocurrents are in good agreement with those measured by AC impedance spectroscopy, which indicates that SPIM is suitable for quantitatively measuring cell impedance.

The LAPS image and $I-V$ curves of different concentrations of yeast were investigated. Three different concentration of yeast $(40 \mathrm{mg} / \mathrm{mL}, 4 \mathrm{mg} / \mathrm{mL}, 0.4 \mathrm{mg} / \mathrm{mL})$ mixed with agarose gel were added onto the same 1,8-nonadiyne modified SOS surface. The $0.4 \mathrm{mg} / \mathrm{mL}$ yeast-gel showed a single layer and a few separate cells under an optical microscope, which did not show a different LAPS signal compared to the blank surface. This may be due to a poor contact between the surface and the cells, as only cells directly in contact with the surface would be visible in the LAPS image. The $4 \mathrm{mg} / \mathrm{mL}$ and $40 \mathrm{mg} / \mathrm{mL}$ yeast-gels showed a clear contrast in the LAPS image at $0.7 \mathrm{~V}$. Compared to the blank surface, the $I-V$ curve of the 4 $\mathrm{mg} / \mathrm{mL}$ yeast-gel showed about $8 \%$ maximum photocurrent decrease and $+40 \mathrm{mV}$ potential shift, while that of the $40 \mathrm{mg} / \mathrm{mL}$ yeast-gel showed about $11 \%$ maximum photocurrent 
decrease and $+75 \mathrm{mV}$ potential shift. The negative surface charge and local impedance increased with the concentration of yeast cells.

\section{Conclusions}

We report LAPS imaging of multilayer yeast cells with good lateral resolution. The LAPS substrate - 1,8-nonadiyne modified SOS - was shown to be insensitive to $\mathrm{pH}$. An agarose gel was used as an effective method for immobilising yeast cells on the sensor surface. The yeast cells caused changes in the local photocurrent that originated from the negative surface charge of the cells and an increase in the local impedance. This shows the potential of LAPS to be developed into a common cell imaging method that can provide more quantitative impedance and charge data than commonly accessible using optical techniques.

In the future, we will adapt this method for single cell LAPS imaging.

\section{Acknowledgements}

The authors thank the EU for providing a Marie Skłodowska-Curie Individual Fellowship (H2020-MSCA-IF-2014-660489) and the China Scholarship Council for providing two PhD studentships (to F.W. and J.W.).

\section{References}

[1] D.J. Stephens, V.J. Allan, Light microscopy techniques for live cell imaging, Science, 300 (2003) 82-86.

[2] K. Nienhaus, G. Ulrich Nienhaus, Fluorescent proteins for live-cell imaging with superresolution, Chem. Soc. Rev., 43 (2014) 1088-1106.

[3] S.A. Jones, S.-H. Shim, J. He, X. Zhuang, Fast, three-dimensional super-resolution imaging of live cells, Nat. Meth., 8 (2011) 499-505.

[4] S. Bergner, P. Vatsyayan, F.-M. Matysik, Recent advances in high resolution scanning electrochemical microscopy of living cells - A review, Anal. Chim. Acta, 775 (2013) 1-13.

[5] I. Morkvenaite-Vilkonciene, A. Ramanaviciene, A. Ramanavicius, 9,10-

Phenanthrenequinone as a redox mediator for the imaging of yeast cells by scanning electrochemical microscopy, Sensors Actuators B: Chem., 228 (2016) 200-206.

[6] H. Yamada, Imaging a single living cell via shear force-based scanning ion conductance microscopy in standing approach mode with differential control, Electrochim. Acta, 136 (2014) 233-239.

[7] J. Seifert, J. Rheinlaender, P. Novak, Y.E. Korchev, T.E. Schäffer, Comparison of atomic force microscopy and scanning ion conductance microscopy for live cell imaging, Langmuir, 31 (2015) 6807-6813.

[8] W. Wang, K. Foley, X. Shan, S. Wang, S. Eaton, V.J. Nagaraj, P. Wiktor, U. Patel, N. Tao, Single cells and intracellular processes studied by a plasmonic-based electrochemical impedance microscopy, Nat. Chem., 3 (2011) 249-255. 
[9] Q. Liu, C. Wu, H. Cai, N. Hu, J. Zhou, P. Wang, Cell-based biosensors and their application in biomedicine, Chem. Rev., 114 (2014) 6423-6461.

[10] D. Hafeman, J. Parce, H. McConnell, Light-addressable potentiometric sensor for biochemical systems, Science, 240 (1988) 1182-1185.

[11] T. Yoshinobu, H. Iwasaki, Y. Ui, K. Furuichi, Y. Ermolenko, Y. Mourzina, T. Wagner, N. Näther, M.J. Schöning, The light-addressable potentiometric sensor for multi-ion sensing and imaging, Methods, 37 (2005) 94-102.

[12] C.F. Werner, C. Krumbe, K. Schumacher, S. Groebel, H. Spelthahn, M. Stellberg, T. Wagner, T. Yoshinobu, T. Selmer, M. Keusgen, M.E.M. Baumann, M.J. Schöning, Determination of the extracellular acidification of Escherichia coli by a light-addressable potentiometric sensor, physica status solidi (a), 208 (2011) 1340-1344.

[13] K.-i. Miyamoto, Y. Hirayama, T. Wagner, M.J. Schöning, T. Yoshinobu, Visualization of enzymatic reaction in a microfluidic channel using chemical imaging sensor, Electrochim. Acta, 113 (2013) 768-772.

[14] P.M. Shaibani, K. Jiang, G. Haghighat, M. Hassanpourfard, H. Etayash, S. Naicker, T. Thundat, The detection of Escherichia coli (E. coli) with the $\mathrm{pH}$ sensitive hydrogel nanofiberlight addressable potentiometric sensor (NF-LAPS), Sensors Actuators B: Chem., 226 (2016) 176-183.

[15] N. Hu, C. Wu, D. Ha, T. Wang, Q. Liu, P. Wang, A novel microphysiometer based on high sensitivity LAPS and microfluidic system for cellular metabolism study and rapid drug screening, Biosens. Bioelectron., 40 (2013) 167-173.

[16] C. Wu, A. Poghossian, T.S. Bronder, M.J. Schöning, Sensing of double-stranded DNA molecules by their intrinsic molecular charge using the light-addressable potentiometric sensor, Sensors Actuators B: Chem., 229 (2016) 506-512.

[17] L. Chen, Y. Zhou, S. Jiang, J. Kunze, P. Schmuki, S. Krause, High resolution LAPS and SPIM, Electrochem. Commun., 12 (2010) 758-760.

[18] J. Wang, Y. Zhou, M. Watkinson, J. Gautrot, S. Krause, High-sensitivity lightaddressable potentiometric sensors using silicon on sapphire functionalized with selfassembled organic monolayers, Sensors Actuators B: Chem., 209 (2015) 230-236.

[19] S. Ciampi, T. Böcking, K.A. Kilian, M. James, J.B. Harper, J.J. Gooding,

Functionalization of acetylene-terminated monolayers on $\mathrm{Si}(100)$ surfaces: a click chemistry approach, Langmuir, 23 (2007) 9320-9329.

[20] J. Wang, F. Wu, M. Watkinson, J. Zhu, S. Krause, "Click" patterning of self-assembled monolayers on hydrogen-terminated silicon surfaces and their characterization using lightaddressable potentiometric sensors, Langmuir, 31 (2015) 9646-9654.

[21] S. Krause, H. Talabani, M. Xu, W. Moritz, J. Griffiths, Scanning photo-induced impedance microscopy - an impedance based imaging technique, Electrochim. Acta, 47 (2002) 2143-2148.

[22] S. Krause, W. Moritz, H. Talabani, M. Xu, A. Sabot, G. Ensell, Scanning photo-induced impedance microscopy - resolution studies and polymer characterization, Electrochim. Acta, 51 (2006) 1423-1430.

[23] J. Wang, I. Campos, F. Wu, J. Zhu, G.B. Sukhorukov, M. Palma, M. Watkinson, S. Krause, The effect of gold nanoparticles on the impedance of microcapsules visualized by scanning photo-induced impedance microscopy Electrochim. Acta, 208 (2016) 39-46.

[24] M. Nakao, S. Inoue, R. Oishi, T. Yoshinobu, H. Iwasaki, Observation of microorganism colonies using a scanning-laser-beam pH-sensing microscope, J. Ferment. Bioeng., 79 (1995) 163-166.

[25] S.M. Tazhibaeva, K.B. Musabekov, A.B. Orazymbetova, A.A. Zhubanova, Surface properties of yeast cells, Colloid Journal, 65 (2003) 122-124. 


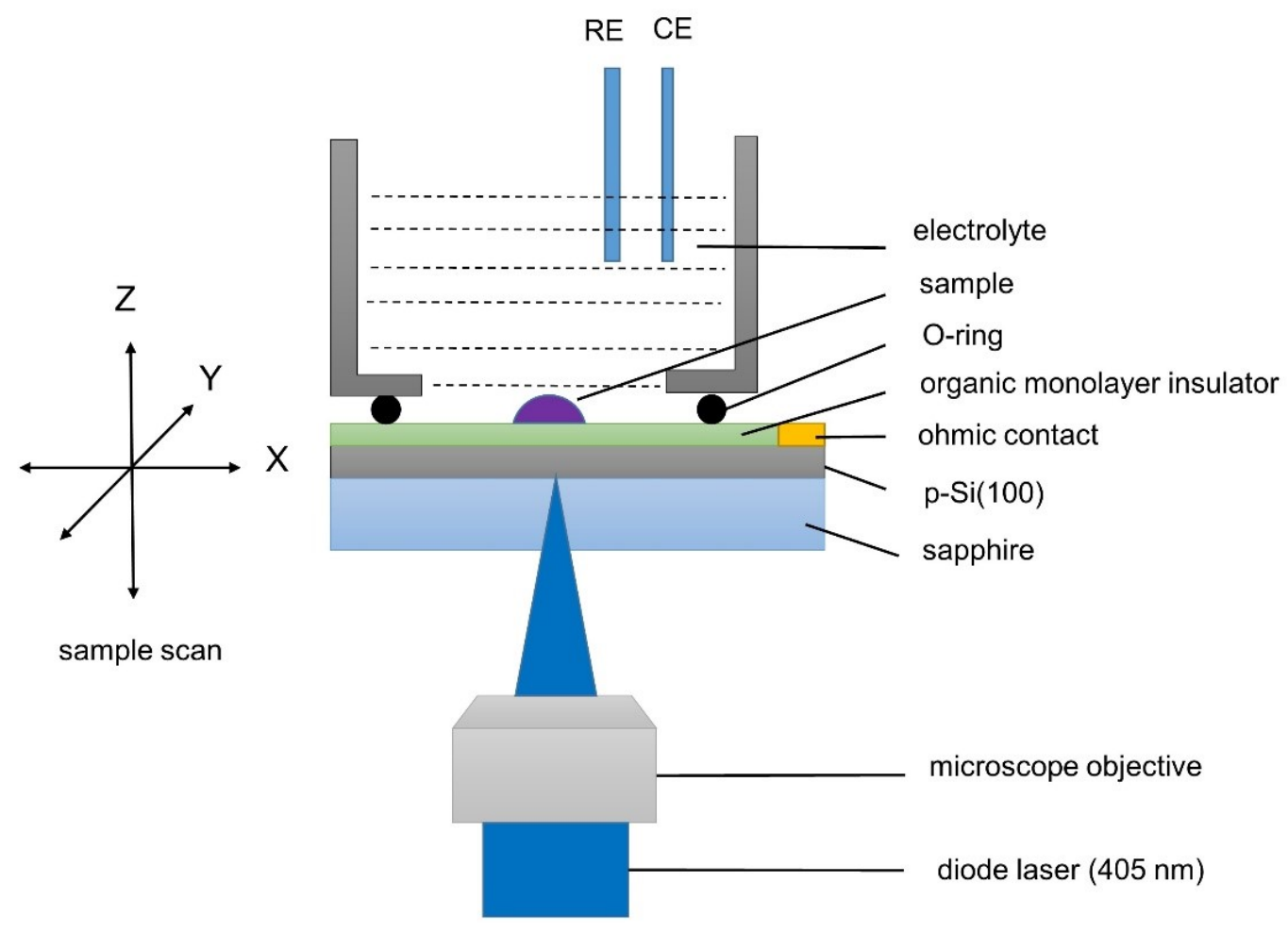

Figure 1. Schematic of the LAPS setup by scanning a focused, modulated laser beam across a silicon-on-sapphire substrate modified with an organic monolayer.

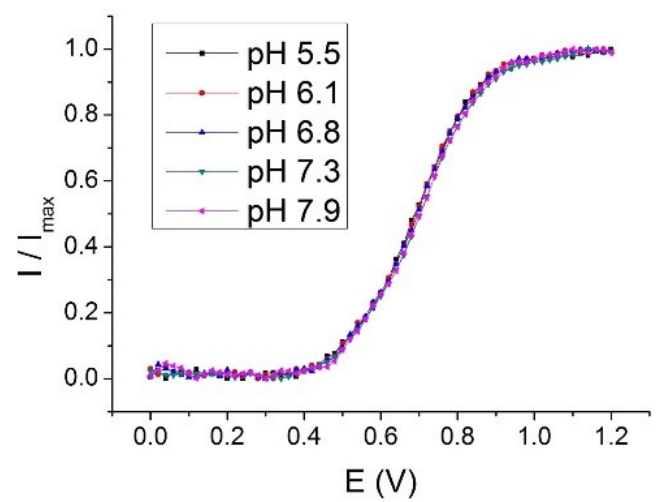

(A)

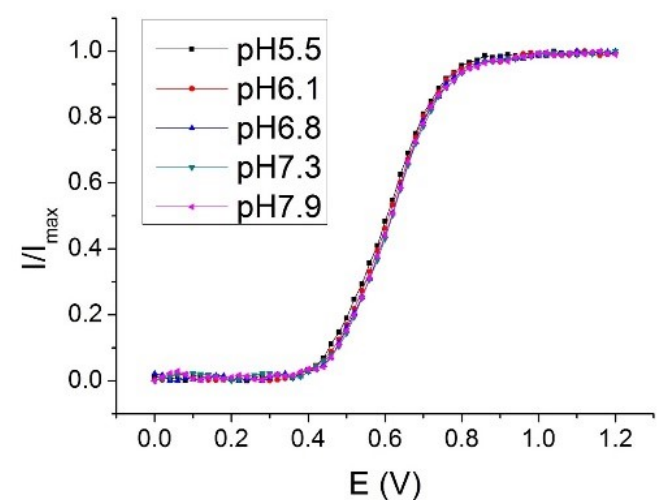

(B)

Figure 2. Normalized I-V curves for a series of different $p H$ values (A) 1,8-nonadiyne modified SOS surface; (B) 1,8-nonadiyne modified SOS surface with agarose gel $\left(I_{\max }=70.0\right.$ $\pm 2.9 n A)$. 
(A)

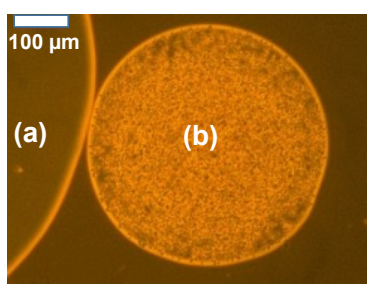

(C)

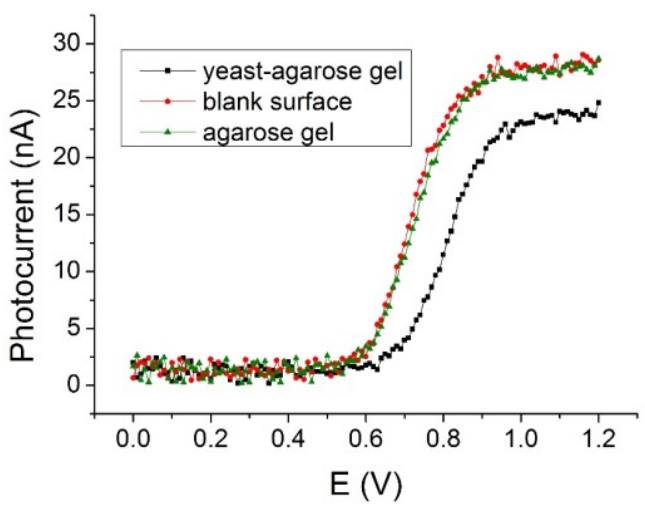

(E)

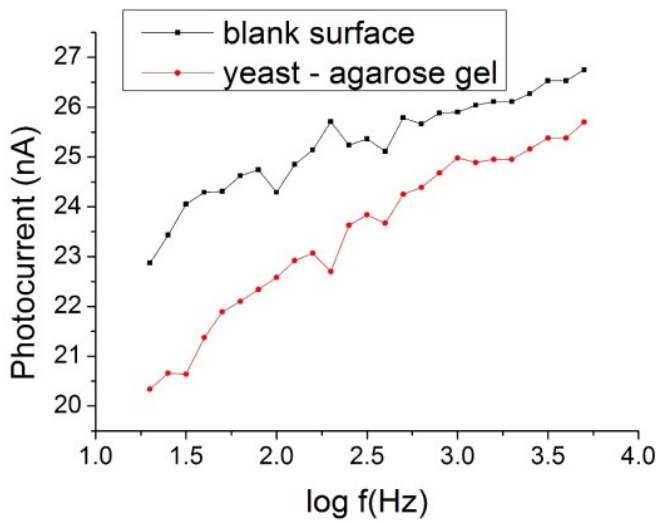

(B)



(D)

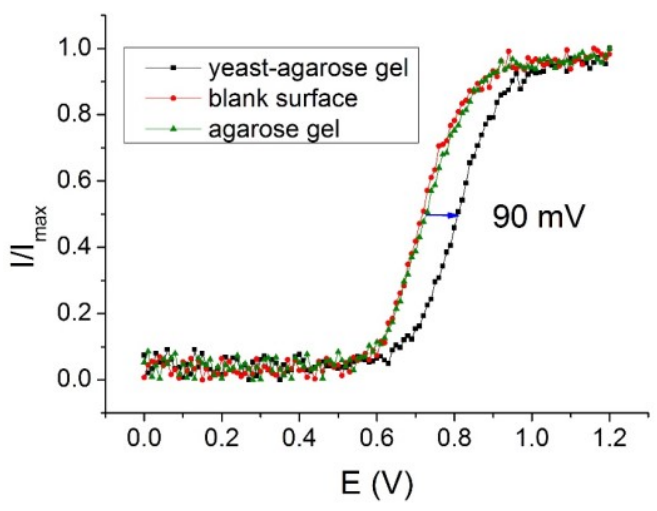

$(\mathrm{F})$

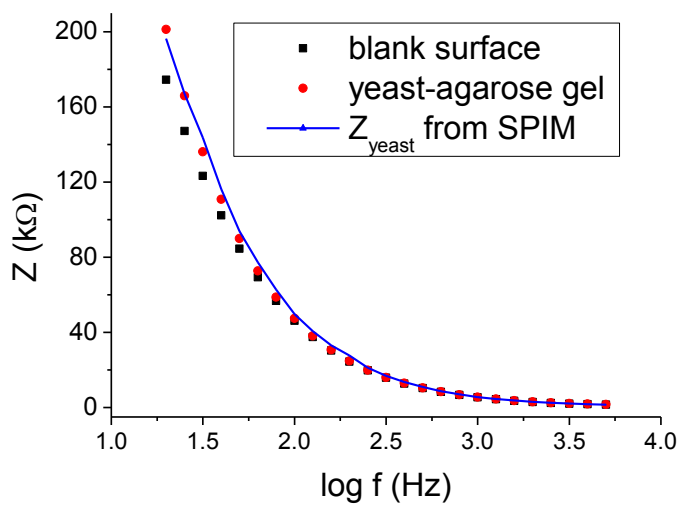

Figure 3. Yeast-agarose gel on a 1,8-nonadiyne modified SOS surface; (A) Optical microscope image of (a) pure agarose gel and (b) yeast-agarose gel; (B) LAPS photocurrent image measured at $0.7 \mathrm{~V}$ (scan range: $700 \mu \mathrm{m} \times 550 \mu \mathrm{m}$, step size: $5 \mu \mathrm{m}$ ) of (a) pure agarose gel and (b) yeast-agarose gel; (C) I-V curves on yeast-agarose gel, blank surface, and agarose gel; (D) normalised I-V curves and potential shift; (E) Maximum photocurrents of the blank surface and yeast modified area on the same surface measured at $1.0 \mathrm{~V}$; (F) Impedance spectra of the entire sample surface before and after immobilization of the yeastagarose gel and $Z_{\text {yeast }}$ calculated from maximum photocurrents of (E) using Equation 2. 